\title{
The risk of acquiring the new influenza $A(H 1 N 1)$ for Brazilian travelers to Chile, Argentina and the USA
}

\author{
Eduardo Massad ${ }^{1,2}{ }^{+}$, Marcelo Nascimento Burattini', Francisco Antonio Coutinho', \\ Cláudio José Struchiner ${ }^{3}$
}

\begin{abstract}
${ }^{1}$ Faculdade de Medicina, Universidade de São Paulo, Rua Teodoro Sampaio 115, 2o andar, 05405-000 São Paulo, SP, Brasil ${ }^{2}$ London School of Hygiene and Tropical Medicine, University of London, London, UK ${ }^{3}$ Programa de Computação Científica-Fiocruz, Rio de Janeiro, RJ, Brasil
\end{abstract}

We estimate the risk of acquiring the new influenza A(H1N1) for Brazilian travelers to Chile, Argentina and the USA. This is done by a mathematical model that quantifies the intensity of transmission of the new virus in those countries and the probability that one individual has of acquiring the influenza depending on the date of arrival and time spent in the area. The maximum estimated risk reached 7.5 cases per 10,000 visitors to Chile, 17 cases per 10,000 travelers to Argentina and 23 cases per 10,000 travelers to the USA. The estimated number of imported cases until 27 July is $57 \pm 9$ from Chile, $136 \pm 27$ from the USA and $301 \pm 21$ from Argentina, which are in accord with the official figures. Estimating the number of imported cases was particularly important for the moment of the disease introduction into this country, but it will certainly be important again as a tool to calculate the number of future imported cases from northern countries in our next inter-epidemic season, were imported cases can constitute again the majority of the new influenza burden to the Brazilian health services.

Key words: influenza A (H1N1) - travelers - risk estimation - mathematical models

The recently emerged swine-origin $\mathrm{H} 1 \mathrm{~N} 1$ influenza viruses are being detected in an increasing number of countries (Neuman et al. 2009). In fact, influenza A viruses have caused several pandemics during the last century and continue to cause annual epidemics (Massad et al. 2008).

The H1N1 flu mainly spreads in the same way as regular "seasonal influenza", being directly transmitted from infected to susceptible individuals through the air from coughs and sneezes or touching infected surfaces.

As of 27 July 2009, 134,503 cases were reported, including 816 deaths (WHO 2009a). The lethality of this new strain varies from $0.11-4.48 \%$ with average around $0.5 \%$ (WHO 2009b).

In Brazil, the last official report from the Ministry of Health (MS 2009) notify 1,566 confirmed cases, the majority $(55.2 \%)$ of which are imported cases. Three countries, Argentina, USA and Chile comprise $91.7 \%$ of the imported cases. However, in 16 July 2009 the country's health authorities declared sustainable transmission of the new influenza virus in Brazil.

One key aspect of planning the control against this new virus strain is the estimation of the risk of acquiring the new influenza people are subject to when traveling to affected areas. This is a composite function of the intensity of transmission in the visited region and the number of visitors to those regions.

Financial support: LIM01-HCFMUSP, CNPq, FAPESP, FAPERJ

+ Corresponding author: edmassad@usp.br

Received 21 August 2009

Accepted 2 February 2010
According to the Brazilian Ministry of Tourism (MT 2009) a total of 7,410 passengers from Argentina, 8,140 from the USA and 3,880 from Chile disembark every day in Brazil. Of these, an estimated 7,264 from Argentina, 6,480 from the USA and 3,340 from Chile are Brazilians. Therefore, a daily total of more than 17,000 Brazilians are at risk of acquiring the new influenza by traveling to those affected countries.

This paper aims to estimate the risk of acquiring the new influenza $\mathrm{A}(\mathrm{H} 1 \mathrm{~N} 1)$ for Brazilian travelers to Chile, Argentina and the USA. This is done by a mathematical model that quantifies the intensity of transmission of the new virus in those countries and the probability that one individual has of acquiring the influenza depending on the data of arrival and time spent in the area.

\section{METHODS}

We applied a mathematical model of SIR type (Massad et al. 2008, Massad \& Wilder-Smith 2009) to calculate the risk (probability) a traveler to an affected area has of acquiring the disease. The model applied is comprised by a system of differential equations with stochastic input components. The model considers two subpopulations representing residents in the affected area and the travelers to the area. The latter subpopulation is followed up along the epidemic course and is used to calculate the probability of acquiring the new influenza strain. We use a deterministic version of the model to describe the influenza dynamics at the resident population level and a stochastic version to describe the risk (probability of contracting influenza) that a single individual traveler visiting the region is subject to. This is based on the assumption that, since the visitors consist of just a small number of individuals, random fluctuations of the contact rate and probability of infection should be considered. Model's details can be found in the appendix. 
The calculated risk is a function of the intensity of transmission, represented by the force of infection (incidence density) (Anderson \& May 1991), the proportion of susceptible travelers with respect to the total population (the travelers are assumed to be distributed uniformly over the transmission area) and the period of time those susceptible travelers remain in the affected area. The model provided a good fit to the observed accumulated number of cases along the period since the beginning of the outbreak.

In order to calculate the probability of an individual acquiring influenza, $\lambda_{f l u}$ after the introduction of a single case in an entirely susceptible population we considered a small susceptible cohort followed through the entire outbreak. The probability of infection in this self-limiting outbreak is then given by the following expression (Massad et al. 2008, Massad \& Wilder-Smith 2009):

$$
\pi_{f l u}=\frac{\int_{0}^{\infty} S^{\prime}(t) \lambda_{f l u}(t) d t}{N^{\prime}(0)}
$$

In the above equation, $S(t)$ and $N^{\prime}(t)$ are respectively the number of susceptible hosts and the total population of the small travelers cohort and $\lambda_{f l u}(t)$ is the force of infection of influenza, defined as the per capita number of new cases per time unit ${ }^{11}$ in the whole (resident) population.

One can also calculate the average risk (probability) of infection for a traveler, who arrives in the affected region at day $\Omega$ after the outbreak is triggered and remains there for $\omega$ days, $\pi_{\text {flu }}^{\text {travelers }}$ :

$$
\pi_{f l u}^{\text {travelers }}=\frac{\int_{\Omega}^{\Omega+\omega} S^{\prime}(t) \lambda_{f l u}(t) d t}{N^{\prime}(\Omega)}
$$

In order to determine which set of parameters best fits the observed data we choose the one that maximizes the likelihood function (Choisy et al. 2007):

$$
L\left(\mathbf{m}(x), \sigma^{2} \mid \mathbf{d}\right)=\prod_{i} \frac{1}{\sqrt{2 \pi \sigma^{2}}} \exp \frac{-\left(\mathbf{d}_{i}-m_{i}\right)^{2}}{2 \sigma^{2}}
$$

where $d$ is a vector of data, $m$ is a vector of model predictions with a mean trend depending on parameter $\mathrm{x}$ and where the errors are assumed to be normally distributed with variance $\sigma 2$. We fixed the error in $20 \%$ (that is, we fixed $\sigma$ such that the confidence interval is equal to $\pm 1.28 \frac{\sigma}{\sqrt{n}}$ and varied the parameter space until (3) was maximized. The seed values from the parameters were taken from Massad et al. (2007).

\section{RESULTS}

In Fig. 1 we show the result of the model (1b) performance in explaining the data from Chile. Note that the model fits the data with reasonable accuracy. The calculated basic reproduction number for this area was 1.78 .

From the model's parameters that best explained the real data we calculated the risk of acquiring flu according to equation (2). For this we assumed that visitors spend one week on average at the affected area. The result is shown in Fig. 2. Note that the maximum risk reached 7.5 $\mathrm{x} 10^{-4}$, that is, 7.5 case per 10,000 visitors.

Figs 3-6 show the results of the same procedures for Argentina and the USA. In this case the model also fits the data with reasonable accuracy. The calculated basic reproduction number for this area was 1.78 . Note that, although the basic reproduction number of Argentina is equal to the Chile's one, the force of infection, the parameter used to calculate the risk, is much higher in this case.

Note that the maximum risk for travelers to Argentina is $1.7 \times 10^{-3}$ that is 1.7 cases per one thousand travelers, more than 20 times the maximum risk for travelers to Chile.

The calculated basic reproduction number for this area was 1.87 . Note that in the case of the USA the fitting of the model to data is not as good as in the previous two cases. This is probably due to the fact that the USA are much more heterogeneous than Chile and

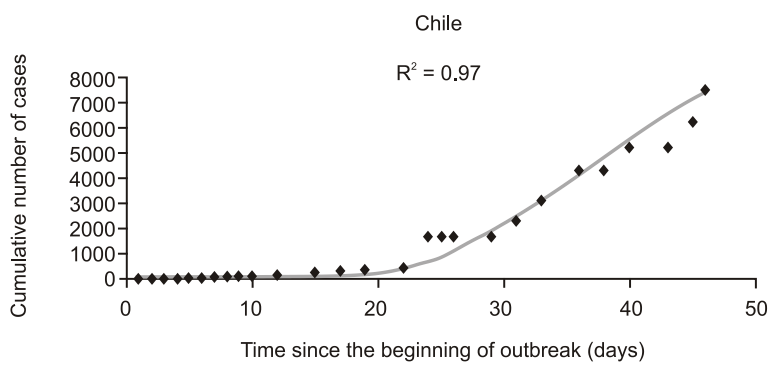

Fig. 1: cumulative number of cases in Chile. Diamonds represent actual data and continuous line the model's output.

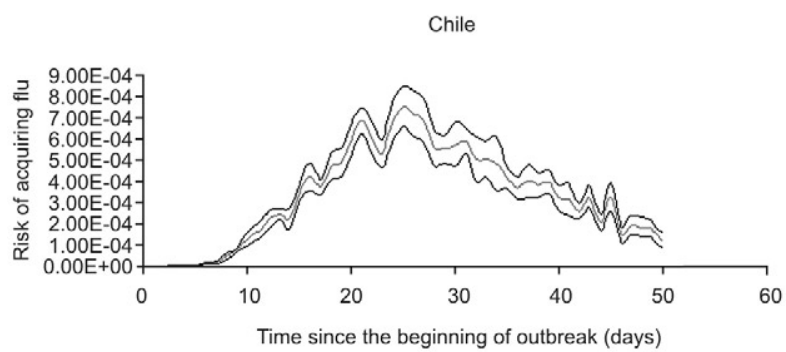

Fig. 2: calculated the risk of acquiring flu for visitors to Chile (average and $95 \%$ confidence interval).

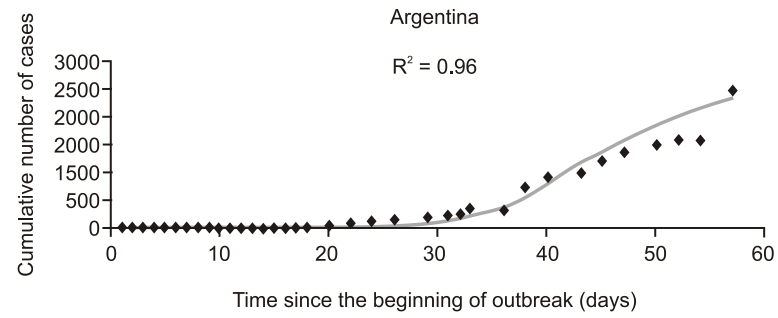

Fig. 3: cumulative number of cases in Argentina. Diamonds represent actual data and continuous line the model's output. 


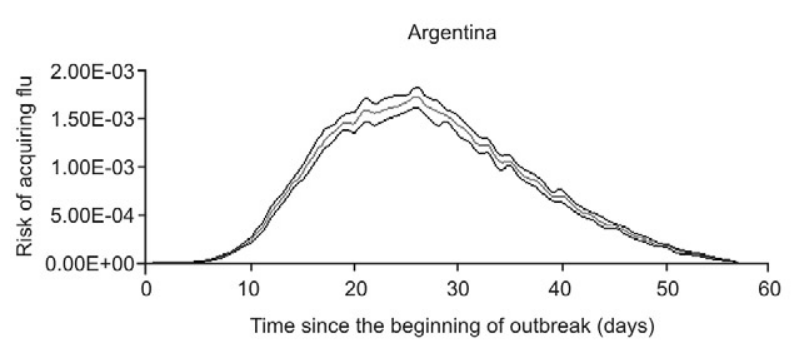

Fig. 4: calculated the risk of acquiring flu for visitors to Argentina (average and $95 \%$ confidence interval).

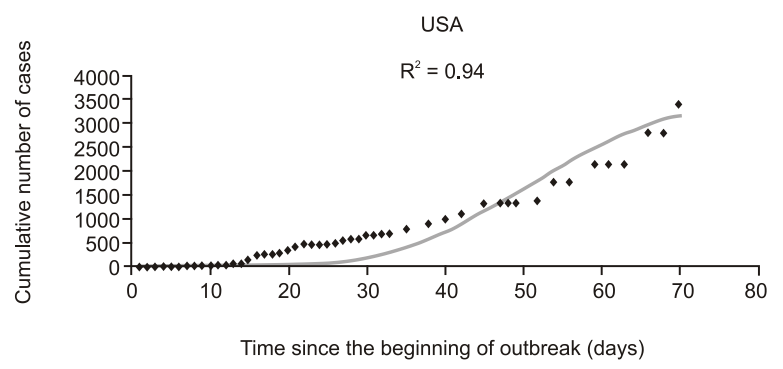

Fig. 5: cumulative number of cases in the USA. Diamonds represent actual data and continuous line the model's output.

USA

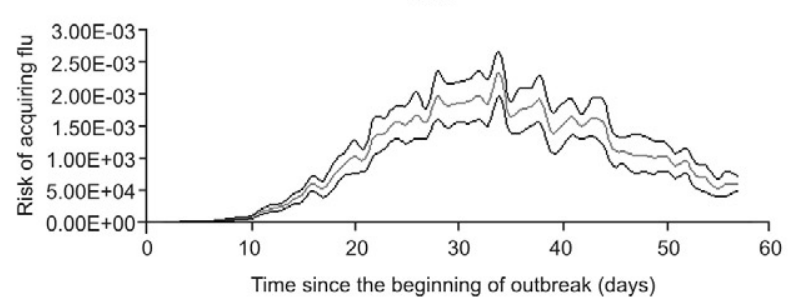

Fig. 6: calculated the risk of acquiring flu for visitors to the USA (average and $95 \%$ confidence interval).

Argentina and the cases are spread throughout a huge geographical area, which contrasts with the very concentrated outbreaks observed in Santiago of Chile or Buenos Aires in Argentina.

In this case the maximum risk is $2.3 \times 10^{-3}$, or 2.3 cases per 1,000 travelers. Although this is the highest risk calculated, it must be weighted by the geographical distribution of Brazilian travelers to the USA, who tend to concentrate their visit in six states, which comprise about $36 \%$ of all American cases. Therefore, Brazilian travelers to the USA are subject to a lower risk than that of those who travel to Argentina but higher than that to Chile.

Next we calculated the expected number of Brazilians that acquired the new influenza by traveling to those areas and spent one week there. For this we multiplied the estimated daily number of Brazilian travelers to each area by the risk of acquiring influenza in one week. The result is shown in Table I, which compares the estimated number of imported cases with the official data (MS 2009). Note that the model is in accord with the observed data from Brazil, although the result for Argentina is not as good as for the other countries analyzed. The possible reasons for this will be discussed below.

\section{DISCUSSION}

As the world faces the first pandemic of the XXI century, each country tries its best to cope with it. Some countries like Mexico (Government of Mexico 2009) impose control measures related to the avoidance of public agglomeration and other measures related to reduce the spread of the virus, other impose draconian measures that include the culling of its entire pig population, like Egypt (BBC 2009).

In Brazil, the measures to reduce the spread of the virus are essentially common sense strategies like the extension of scholar holidays and the education of the population for frequent hands cleansing and to avoid agglomerations in public places. The impact of the governmental attempts on the virus spread is difficult to estimate and the number of cases and deaths continues to pile up.

Although Brazil has already sustained circulation of H1N1 the majority of cases are still imported (as of 27 July 2009), in particular from the countries analyzed in this paper.

As can be observed in the three curves related to risk of acquiring influenza for travelers, the worst situation is already over and except for the USA there is only residual risk to get the infection for those traveling nowadays to the other areas analyzed. However, it is important to emphasize the significance of our findings. The calculation of the risk to get influenza for travelers can be used to contrast the probability of infection with the age-dependent incidence of infection in actual travelers. This would allow the estimation of relative risks distributed according to age and raise hypotheses on why some ages are more affected than others.

As for our results, the inspection of Figs 1, 3 and 5 demonstrates that the model explains the observed data with good accuracy and that the parameters chosen are reliable enough to estimate the risks shown in Figs 2, 4 and 6 . For the case of travelers to the USA, if we multiply the number of travelers by the estimated risk to acquire the flu, the number of expected cases would be about three times the one presented in Table I. How-

\section{TABLE I}

Comparison between the model's outcome and the official data

\begin{tabular}{lcc}
\hline & $\begin{array}{c}\text { Estimated number } \\
\text { of imported cases }\end{array}$ & $\begin{array}{c}\text { Official number of } \\
\text { imported cases (as } \\
\text { 15 July 2009) }\end{array}$ \\
\hline Chile & $57 \pm 9$ & 70 \\
USA & $136 \pm 27$ & 109 \\
Argentina & $301 \pm 21$ & 416 \\
\hline
\end{tabular}


ever, as mentioned above, Brazilian travelers tend to concentrate their visited places in six American states, which comprise for around $36 \%$ of the total number of cases. Therefore, as the risk calculated is for the case of travelers to any place randomly chosen, the actual number of imported cases from the USA must be adjusted for that percentage.

The results of the estimated number of imported cases shown in Table I demonstrate that the calculated risks allowed a good retrieving of the reported cases for Chile and the USA. However, the model diverges from the actual data in the case of Argentina. This is because in the case of that country there is a substantial number of travelers who go to Argentina by car and who are not computed in the official statistics available. Therefore, the number of visitors to Argentina used in our calculations is certainly an underestimation of the actual number of visitors. This could explain the divergence of the model from the real data in the case of Argentina. The calculated basic reproduction numbers for the three countries analyzed are similar among themselves and are in accord to the current estimations for the new influenza virus (Fraser et al. 2009).

With the exception of the USA it should be expected that the number of new cases of influenza $\mathrm{A}(\mathrm{H} 1 \mathrm{~N} 1)$ in the studied countries starts to subside from now on and so the correspondent risk for travelers to those places should be proportionately reduced. However, the actual behavior of this new virus in the next autumn/winter in the Northern Hemisphere is still to be known. Only then can we estimate the risk for Brazilian travelers to the USA from now on with better accuracy.

The model proposed in this paper provides a way to estimate the number of imported cases of the new influenza $\mathrm{A}(\mathrm{H} 1 \mathrm{~N} 1)$ to Brazil. This was particularly important for the moment of the disease introduction into this country, but it will certainly be important again as a tool to calculate the number of future imported cases from northern countries in our next inter-epidemic season, were imported cases can constitute again the majority of the new influenza burden to the Brazilian health services.

The model - The model assumes that the population of humans is divided into three compartments summarized in Table II and described below. In addition, we separate from the human general population (individuals that re-

\section{TABLE II}

Models' variables

\begin{tabular}{lc}
\hline$S$ & Human susceptible individuals in the "probe" \\
$I$ & Human infected individuals in the "probe" \\
$R^{\prime}$ & Human recovered individuals in the "probe" \\
$N^{\prime}$ & $S+I+R^{\prime}$ \\
$S$ & Human susceptible individuals in the resident population \\
$I$ & Human infected individuals in the resident population \\
$R$ & Human recovered individuals in the resident population \\
$N$ & $S+I+R$ \\
\hline
\end{tabular}

side in the area) a small cohort (Massad et al. 2008) denoted by primes, followed through their entire exposure to calculate the risk of influenza acquisition. The model's variables are shown in Table I.

The model's dynamics is described by the following set of equations:

$$
\begin{aligned}
& \frac{d S^{\prime}}{d t}=-\beta^{\prime} S^{\prime} \frac{\left(I+I^{\prime}\right)}{\left(N+N^{\prime}\right)}-\mu S^{\prime} \\
& \frac{d I^{\prime}}{d t}=\beta^{\prime} S^{\prime} \frac{\left(I+I^{\prime}\right)}{\left(N+N^{\prime}\right)}-(\mu+\gamma+\alpha) I^{\prime} \\
& \frac{d R^{\prime}}{d t}=\gamma I^{\prime}-\mu R^{\prime}
\end{aligned}
$$

$$
\frac{d S}{d t}=-\beta S \frac{\left(I+I^{\prime}\right)}{\left(N+N^{\prime}\right)}-\mu S+r\left(S+S^{\prime}+R+R^{\prime}\right)\left(1-\frac{N+N^{\prime}}{\mathrm{K}}\right)
$$$$
\frac{d I}{d t}=\beta S \frac{\left(I+I^{\prime}\right)}{\left(N+N^{\prime}\right)}-(\mu+\gamma+\alpha) I
$$

$$
\frac{d R}{d t}=\gamma I-\mu R
$$

In the main text we used

$$
\lambda_{f l u}=\beta \frac{\left(I+I^{\prime}\right)}{\left(N+N^{\prime}\right)}
$$

The model's parameters, their biological meaning and values used in the simulations are shown in Table III.

The equations (1b) when summed term by term with equations (1a) with $\beta^{\prime}=\beta$ represent a classical SIR model for infectious diseases as given by, for example, in Anderson and May (1991). Equations (1a) represent a cohort that was separated from the main population with the purpose of calculating the probabilities needed. Note that there are no entrance terms to the equations (1a) and that infected and newborns individuals resulting from them are added to the general population represented by equations (1b). A prime was added to the contact rate of the small cohort represented by equations (1a) because in the simulations we let it to be a random variable. In fact $\beta$ ' in the simulations was taken to be Poisson distributed with the average equal to the term $\beta$ for the general population represented by equations (1b). This is based on the assumption that any single individual (and hence an occasional visitor) suffers a random number of potentially infective contacts with infective individuals. Since the cohort was very small with respect to the main population the fact that we took $\beta$ ' to be a random variable had no effect on the dynamics of the main population.

Control measures from a given point in time were simulated by suddenly [from day $v_{i}$ since the beginning of the outbreak, $i=$ Chile (27), Argentina (27), USA (20)] 


\section{TABLE III}

Model's parameters

\begin{tabular}{lcr}
\hline$\beta_{\text {Chile }}$ & Potentially infective contacts - Chile & 0.54 days $^{-1}$ \\
$\beta_{\text {Argentina }}$ & Potentially infective contacts - Argentina & 0.53 days $^{-1}$ \\
$\beta_{\text {USA }}$ & Potentially infective contacts - USA & 0.57 days $^{-1}$ \\
$\beta^{\prime}$ & Poisson $(\beta)$ & variable $^{-1}$ \\
$\mu$ & Natural mortality rate & $5.50 \times 10^{-5}$ days $^{-1}$ \\
$\alpha$ & Additional mortality induced by the disease & 0.02 days $^{-1}$ \\
$\gamma$ & Recovery rate from disease & 0.29 days $^{-1}$ \\
$r$ & Birth rate & 0.03 days $^{-1}$ \\
$\kappa$ & Carrying capacity of the population & $50 \times 10^{6}$ \\
\hline
\end{tabular}

letting both $\beta$ and $\beta$ ' decreasing linearly with time, that is, we made:

$$
\begin{aligned}
& \beta=\beta_{i}-\left(\varphi_{i} t\right) \theta\left(t-v_{i}\right) \\
& \beta^{\prime}=\operatorname{Poisson}\left(\beta_{i}-\left(\varphi_{i} t\right) \theta\left(t-v_{i}\right)\right.
\end{aligned}
$$

where $\varphi_{i}[i=$ Chile (0.0057), Argentina (0.0057), USA $(0.005)]$ is an attenuation factor and $\theta\left(t-v_{i}\right)$ is the Heaviside function.

We assumed a Poisson distribution for the parameter $\beta$ ' based on the fact that this is a composite parameter comprising potentially infective contacts (a discrete random variable) and the probability that those contacts will generate a new infection.

\section{REFERENCES}

Anderson RM, May RM 1991. Infectious diseases in humans, Oxford University Press, Oxford, $757 \mathrm{pp}$.

BBC - British Broadcasting Corporation 2009. [homepage on the Internet]. [accessed 24 April, 2009]. Available from http://news. bbc.co.uk $/ 2 / \mathrm{hi} / \mathrm{middle}$ east $/ 8024946$.stm.

Choisy M, Guégan JF, Rohani P 2007. Mathematical modeling of infectious diseases dynamics. In M Tibayrene, Encyclopedia of infectious diseases: modern methodologies, John Wiley and Sons Inc, Hoboken, p. 379-404.

Fraser C, Donnelly CA, Cauchemez S, Hanage WP, Van Kerkhove MD, Hollingsworth TD, Griffin J, Baggaley RF, Jenkins HE, Lyons EJ, Jombart T, Hinsley WR, Grassly NC, Balloux F, Ghani AC, Ferguson NM, Rambaut A, Pybus OG, Lopez-Gatell H, Alpuche-Aranda CM, Chapela IB, Zavala EP, Guevara DME, Chec- chi F, Garcia E, Hugonnet S, Roth C 2009. Pandemic potential of a strain of influenza $\mathrm{A}(\mathrm{H} 1 \mathrm{~N} 1)$ : early findings. Science 324 : $1557-1561$

Government of Mexico 2009. [homepage on the Internet]. [accessed 12 June 2009]. Available from: http://www.vacationparadise. com/traveltips/tipsmexicol.htm.

Massad E, Burattini MN, Coutinho FAB 2007. The 1918 influenza A epidemic in the city of São Paulo, Brazil. Med Hypotheses 68: 442-445.

Massad E, Ma S, Burattini MN, Tun Y, Coutinho FAB, Ang LW 2008. The risk of chikungunya fever in a dengue-endemic area. J Travel Med 15: 147-155.

Massad E, Wilder-Smith A 2009. Risk estimates of dengue in travelers to dengue endemic areas using mathematical models. J Travel Med 16: 191-193.

MS - Ministério da Saúde do Brasil 2009. [homepage on the Internet]. [accessed 27 July 2009]. Available from: http://portal. saude.gov.br/portal/arquivos/pdf/boletim epidemiologico influenza_23_09_2009.pdf.

MT - Ministério do Turismo do Brasil 2009. [homepage on the Internet]. [accessed 27 July 2009]. Available from: http://www.turismo.gov.br/turismo/o ministerio/publicacoes/.

Neumann G, Noda T, Kawaoka Y 2009. Emergence and pandemic potential of swine-origin H1N1 influenza virus. Nature 459: 931-939.

WHO - Word Health Organization 2009a. [homepage on the Internet]. [accessed 27 July 2009]. Available from: http://www.who.int/csr/ don/2009_07_27/en/index.html.

WHO - Word Health Organization 2009b. [homepage on the Internet]. [accessed 27 July 2009]. Available from: http://www.who.int/en/. 